\title{
Auditory temporal resolution in adaptive tasks
}

\author{
Gap detection investigation
}

Abdulsalam A. Alhaidary, PhD, Kishore Tanniru, MSc, Adel F. Aljadaan, PhD, Lamya M. Alabdulkarim, PhD.

\begin{abstract}

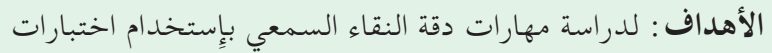

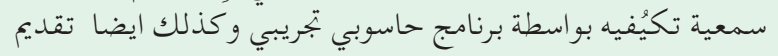

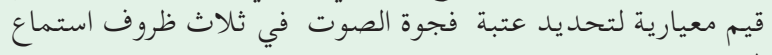

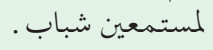

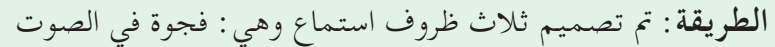

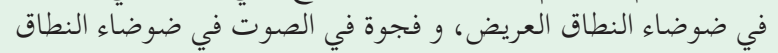

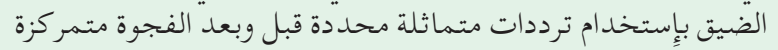

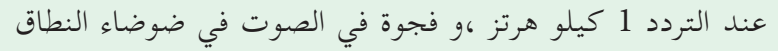

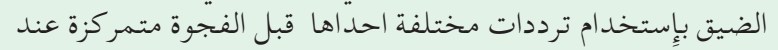

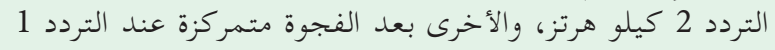

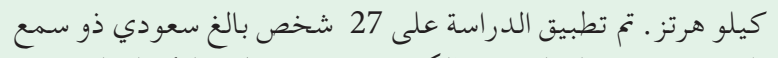

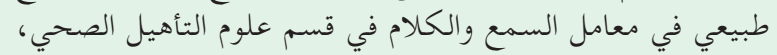

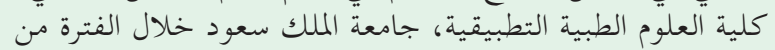

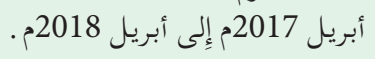

النتائج: وجد أن متوسط الأداء في تحديد الفجوة في الصوت هي العي

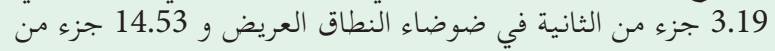

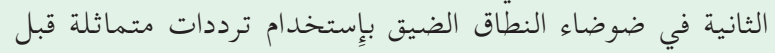

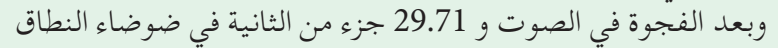

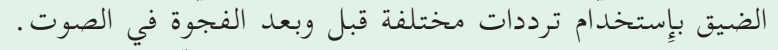

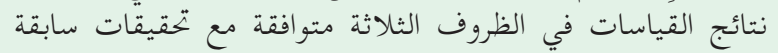

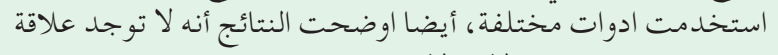
ارتباط بين الأداء في حالات الإِستماع الثلاثة .

الحخاتمة: أظهرت الدراسة الحالية أن البرنامج التجريبي هو أداة يمكن

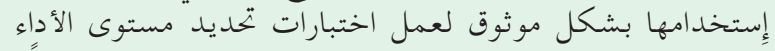

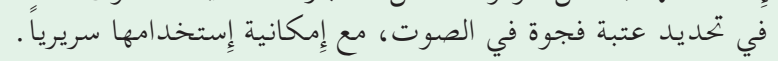

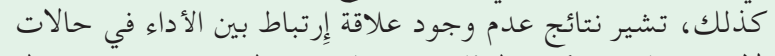

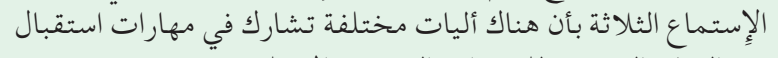
دقة النقاء السمعي للمثيرات السمعية المختلفة . لمنئة
\end{abstract}

Objectives: To study auditory temporal resolution skills using adaptive auditory tasks designed with a computer-based experimental program, and to provide normative values for gap detection thresholds (GDTs) of young listeners in 3 listening conditions.

Methods: The GDTs were established under 3 stimulus conditions: 1) broadband noise (BBN), 2) narrowband noise within-channel (NBN WC) using similar leading and trailing markers centered at $1.0 \mathrm{KHz}$, and 3) narrowband noise across-channel (NBN AC) with the leading marker centered at $2.0 \mathrm{KHz}$ and the trailing marker centered at $1.0 \mathrm{KHz}$. In within-subjects design, the GDTs were obtained from 27 normal hearing young Saudi adults at Speech and Hearing Laboratories, Department of Rehabilitation Health Sciences, College of Applied Medical Sciences, King Saud University, Riyadh, Saudi Arabia between April 2017 and April 2018.

Results: The mean GDTs for the BBN condition was 3.19 millisecsond (msec), NBN WC was $14.53 \mathrm{msec}$, and NBN AC was $29.71 \mathrm{msec}$. Our findings for the GDTs measured in the 3 conditions were consistent with those of earlier investigations that used different instrumentations. Also, no correlations among the GDTs of the 3 stimulus conditions were found.

Conclusion: The present study showed that experimental program is a reliable tool with clinical potential to estimate GDTs across different conditions. Also, the findings of no correlations in the GDTs across stimulus conditions suggest that different processes were involved in the perception of the temporal gap for different stimulus conditions.

Saudi Med J 2019; Vol. 40 (1): 52-58 doi: 10.15537/smj.2019.1.23814

From the Department of Rehabilitation Sciences, King Saud University, Riyadh, Kingdom of Saudi Arabia.

Received 30th August 2018. Accepted 5th December 2018.

Address correspondence and reprint request to: Dr. Abdulsalam Alhaidary, College of Applied Medical Sciences, King Saud University, Riyadh, Kingdom of Saudi Arabia.E-mail: aalhaidary@ksu.edu.sa ORCID ID: orcid.org/0000-0002-2052-5184 
$\mathrm{F}$ or a listener, a clear understanding of speech requires many intact auditory processes. Study has shown that temporal processing skills, the regulation and monitoring of changes in sound energy over time, are essential to this understanding. Auditory temporal processing is classified into different categories or abilities, such as temporal integration (summation of energy over time), temporal discrimination (differentiating 2 acoustic events solely by duration), temporal masking (the effect of the decreased audibility of a sound due to a preceding or following sound), temporal ordering (perception fluctuations in intensity and frequency over time), and temporal resolution/acuity (recognition of temporal cues in acoustic energy as the means to differentiate acoustic signals). ${ }^{1}$ With regard to measuring temporal resolution/acuity, extensive studies have reported on temporal gap detection, duration discrimination, temporal integration, and temporal modulation transfer functions. ${ }^{2}$ Scholars have used different procedures to investigate these abilities, such as adaptive tests of temporal resolution, ${ }^{3}$ gap-in-noise (GIN) test, ${ }^{4}$ random gap detection test, ${ }^{5}$ and gap detection testing using MATLAB, MathWorks, Inc. (The Math Works, Natick, MA, USA). Perceptual deficits in temporal resolution skills or other central auditory processing skills such as sound localization, lateralization, and discrimination may cause central auditory processing disorder (CAPD). ${ }^{1}$ Temporal gap test is one of the clinical test battery for diagnosing CAPD. The present study reports Saudi data on gap detection thresholds (GDTs) by using a Windows-based psychoacoustic experiment program named Psycon developed by Kwon. ${ }^{6}$

The GDT is a temporal gap detection test involves determining the minimum silence duration embedded in a signal that can be detected by a listener. Extensive study was conducted to identify the GDT for broadband noise $(\mathrm{BBN})$ and narrowband noise $(\mathrm{NBN})$. Measurements of the GDT using NBN sounds are commonly carried out using within-channel (WC) gap detection (similar markers before and after the gap) or across-channel (AC) gap detection (dissimilar leading and trailing markers before and after the gap). Studies on gap detection with normal hearing adult listeners have shown that the lowest gap duration that has been detected for BBN sound was in the range of 2 to 5 millisecond (msec). ${ }^{7-10}$ High GDTs have been reported using NBN sounds in the range of 3 to 7

Disclosure. Authors have no conflict of interests, and the work was not supported or funded by any drug company. msec for the WC paradigm. ${ }^{8,10,11}$ Also, the gap detection performance for WC testing varies as a function of frequency. For example, Florentine et $\mathrm{al}^{12}$ have found psychometric functions for gap detection using band pass noises with center frequencies between $0.25 \mathrm{KHz}$ to $8.0 \mathrm{KHz}$. Their results suggest that the GDT varied between $4.6 \mathrm{msec}$ for $8.0 \mathrm{KHz}$ band pass noise to 88.1 msec for $0.25 \mathrm{KHz}$ band pass noise. Furthermore, the study consistently reported a higher GDT when using an AC gap detection paradigm. ${ }^{10,11,13-17}$ For example, Florentine et $\mathrm{al}^{16}$ reported a GDT of approximately 40-42.6 msec for AC NBN 2-1 KHz msec for young adults with normal hearing.

Lister et $\mathrm{al}^{3}$ have proposed a gap detection test that uses a computerized software application named adaptive tests of temporal resolution (ATTR). The experimental procedures of ATTR were applied into more clinically used procedures for determining the GDT.8,10,11 The ATTR software requires a computer with a sound card. It uses sets of stimuli that are stored offline as a waveform audio file format with fixed silence intervals. ${ }^{10}$ In addition, the experimental procedures are pre-determined in the ATTR platform with limited flexibility to investigate the cause and effect relation of stimulus-related parameters. As an alternative to ATTR, Kwon ${ }^{6}$ has described an independent scripting language device called auditory syntax (AUX) that was developed to generate and process audio signals using relatively simple codes and could be used as experimental platform for temporal gap detection tasks. The scripting application software package includes details for writing scripts to create psycho-perceptual tasks. In addition, Kwon $^{6}$ designed a Windows-based psychoacoustic experiment program named Psycon that uses these AUX scripting syntax to create and process auditory signals. There is a need to explore such computer applications for their feasibility and reliability for assessing gap detection abilities, and for their clinical utility.

The aim the study is to describe the procedures for determining temporal resolution tasks using a Windows-based psychoacoustic experiment program named Psycon as described by Kwon. ${ }^{6}$ Psycon (Version 2.18) manipulates complex signal parameters in a relatively easy manner, and to systematically observe cause and effect relations in a consistent manner. The present study has used the clinical potential of Psycon to establish the normative values for the GDTs of normal hearing listeners both within and across a channel paradigm.

Methods. Twenty-seven native Arabic male speakers with normal hearing sensitivity volunteered to participate in the study. We used within-subject study experimental design in which all participants performed 
all experimental conditions. We screened all participants for normal hearing abilities based on an inclusion criteria of air conduction pure tone thresholds less than or equal to $15 \mathrm{~dB}$ (for octave frequencies between 0.25 to $8.0 \mathrm{KHz}$ ). All participants had normal findings on an otoscopic examination, and $\mathrm{A} / \mathrm{As}$ tympanograms with acoustics reflex present at $90 \mathrm{~dB}$ HL. All participants were undergraduate students from the College of Applied Medical Sciences, King Saud University, Riydah, KSA and they were blinded to the procedure used in the study. The ages of the participants ranged from 21.08 to 24.44 years $(22.21 \pm 0.73)$. The case histories of all the participants included in the study-regarding their history of middle ear infection, cognitive illness, head trauma, or noise exposure-were unremarkable. All the experimental procedures were carried out at the speech and hearing laboratories, Department of Rehabilitation Health Sciences, College of Applied Medical Sciences, King Saud University, Riyadh, KSA between April 2017 to April 2018. All the study procedures followed the ethical guidelines of the Research Ethics Committee of the College of Applied Medical Sciences, King Saud University, Riyadh, Saudi Arabia.

A 2 channel diagnostic audiometer (Grason Stadler GSI-61TM (GSI-61TM, Eden Prairie, Minnesota, USA) for hearing assessment and to route the stimuli in the experimental procedures were used. Supra-aural headphones TDH-50P with a mod 51 ear cushion were used to deliver the stimuli for the study. A clinical otoscope and calibrated GSI-Tympstar were used to assess the participants' middle ear functions prior to their participation in the experiment. All the procedures were carried out in the subject room of an audiometric test booth (model: RS142 acoustic systems). Psycon (Version 2.18) was used to generate the desired experimental stimulus and to collect the participants' responses. ${ }^{6}$ Psycon is a Windows-based psychoacoustic experimental program available under Academic Free License 3.0. The program was operated by using commands scripted with the auditory syntax (AUX) detailed in the program manual. We installed and ran the program distributed under the Academic Free License (Version 3.0) on a Hawlett-Packard Compaq nx7300 laptop with a standard built-in sound card (Intel).

All the gap detection measures required the participant to listen to 2 stimuli presented successively; a standard (no-gap) stimulus and an adjustable (gap-in) stimulus. A sampling frequency of $44.1 \mathrm{KHz}$ and an amplitude factor of $-5 \mathrm{~dB}$ (to avoid distortion at peak level) was used to generate all the stimuli. We presented the stimuli monaurally and at a constant level of 60 $\mathrm{dB}$ HL (volume unit meter was set to 0 ). The desired stimulus under 3 categories: BBN GDT, NBN WC GDT, and NBN AC GDT were generated.

To measure the GDT using BBN, the Gaussian noise was used without any edge filters. Both the standard and adjustable stimuli under BBN GDT were $500 \mathrm{msec}$ with a 10 -msec rise time at the start of the signal and a 10 -msec fall time at the end of the signal. The gap (silence) duration around the center of the signal (silence inserted around $250 \mathrm{msec}$ ) were symmetrically inserted and maintained the total signal duration as $500 \mathrm{msec}$. Spectrograms of the no-gap stimulus and the gap-in stimulus with a $30 \mathrm{msec}$ silence interval are represented in Figure 1. The adjustable stimulus depicted in Figure 1 illustrate that the gap duration was from $235 \mathrm{msec}$ to $265 \mathrm{msec}$ (symmetrical around $250 \mathrm{msec}$ ). Figure 2 depicts the long-term average spectrum of the signals generated for BBN GDT. All the spectrograms and long-term average spectrum measures were obtained through Praat Version 6.0.14..$^{18}$

The NBN WC GDT and NBN AC GDT gap detection measures, narrow band noises were instantly
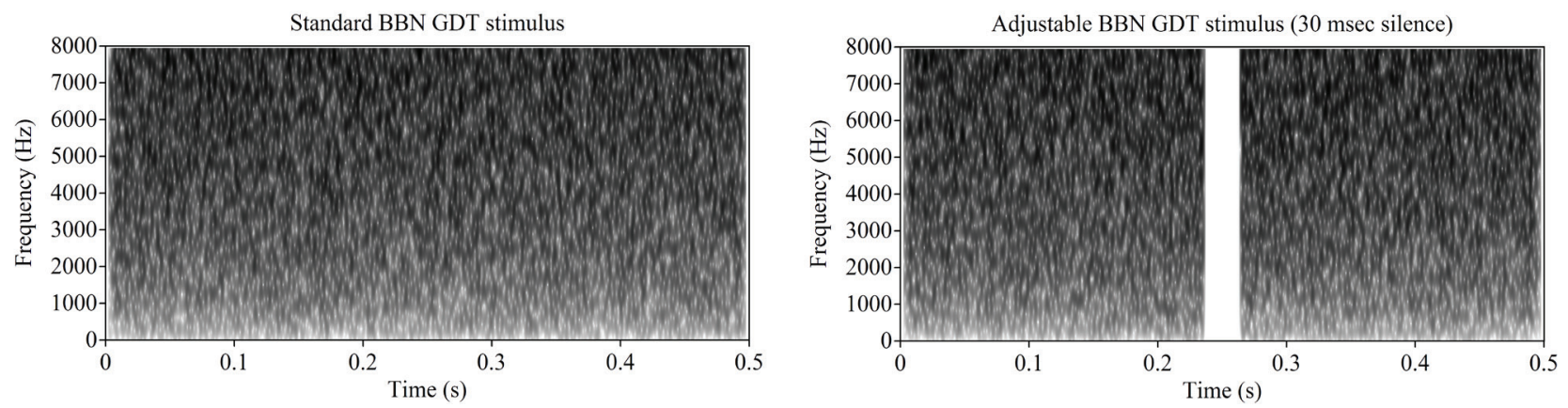

Figure 1 - Spectrograms for no-gap stimulus (left side) and the gap-in stimulus. As an example a 30 msec silence interval was illustrated in gap-in stimulus (right side). BBN - broadband noise, GDT - gap detection thresholds 
generated from Gaussian noise with each $1 / 4^{\text {th }}$ octave bandwidth and frequency geometrically centered at $1.0 \mathrm{KHz}$ or $2.0 \mathrm{KHz}$ with an $8^{\text {th }}$ order Butterworth filter (pass band ripple as $0.5 \mathrm{~dB}$ and stop band attenuation $-40 \mathrm{~dB}$ ). Both the leading (before the gap) and trailer (after the gap) markers were $1.0 \mathrm{KHz}$ centered NBN in all stimuli of the NBN WC GDT, and for NBN AC GDT, the leading marker was always $2.0 \mathrm{KHz}$ centered NBN, and trailer marker was $1.0 \mathrm{KHz}$ centered NBN. The duration of the leading marker was always constant at $300 \mathrm{msec}$, and the duration of the and the duration of the trailing marker varied randomly between 250 to $350 \mathrm{msec}$ per each trial in each experimental run. The trailer maker duration was randomized to avoid the detrimental effects of constant overall stimulus duration. ${ }^{10,19,20}$ The rise time of the leading marker and the fall time of the trailer maker were ramped with 10 msec. However, the offset of the leading marker and the onset of the trailer marker were one msec. These one msec transients ensured similar gating properties in both the standard and test intervals. ${ }^{9,14}$ These transients are illustrated in Figure 3.

Each participants seated in the subject room of the audiometric booth facing a laptop monitor and provided them with TDH-50P Supra-aural headphones with a mod 51 ear cushion that were connected to a GSI-61 $1^{\mathrm{TM}}$ 2 channel clinical audiometer. Before beginning the experiment, all the participants were provided with detailed instructions along with a visual depiction of the screen shots of the experiment. The listeners were asked to select one sound that had a longer silence in it from the 2 sounds heard one after the other. A corresponding block was highlighted on the screen with each sound presentation. A wireless keyboard was connected to

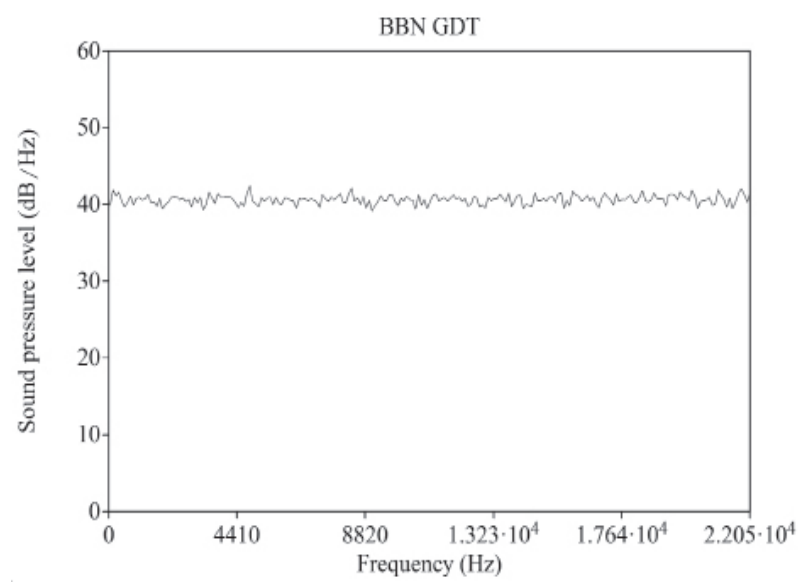

Figure 2 - Long-term average spectrum of the broad band noise used for estimation of gap detection threshold (BBN GDT) stimulus. $\mathrm{Hz}=$ hertz and $\mathrm{dB}=$ decibels . the laptop and handed to the participant to select the desired interval by pressing a numerical key (1 or 2 ) after listening to both the standard and adjustable stimuli. Visual feedback on the correctness of their selection was displayed after each desired selection for each trial. A trail test condition was used involving 15 trials to check all the participants' understanding of the instructions and their familiarization with the task.

Throughout the experimental procedures, an inter-stimulus interval of $500 \mathrm{msec}^{14,21}$ and a minimum $1000 \mathrm{msec}$ interval were used after each subject response before presenting the next set of stimuli. We randomly assigned the stimulus conditions, and adoptively changed the imposed gap duration of the adjustable stimulus, based on the participants' response. If the stimulus condition was $\mathrm{BBN}$, the gap for the adjustable stimulus started at $15-\mathrm{msec}$, and if the stimulus condition was NBN WC or NBN AC, the gap duration for the adjustable stimulus started at 50 -msec. The classic Levitt's adaptive 2-down 1-up procedure was utilized using a 2 interval alternative force choice method to target the $70.7 \%$ correct gap detection threshold. ${ }^{22}$ To ensure fine measurements for the first 5 reversals of each experimental run or trial, the step size was 1.2 factors, and for the next 6 reversals, the step size was 1.05 factors.

An average of the last 4 reversals out of 11 reversals were used to compute the GDTs so to reduce the variation in the considered GDT. ${ }^{14}$ We computed an individual standard deviation for each experimental run from the last 4 reversal points to determine whether the GDT measure with that condition needed to be repeated (when the SD was greater than $5 \mathrm{msec}$ ). Each participant's response for all stimuli trials were saved,
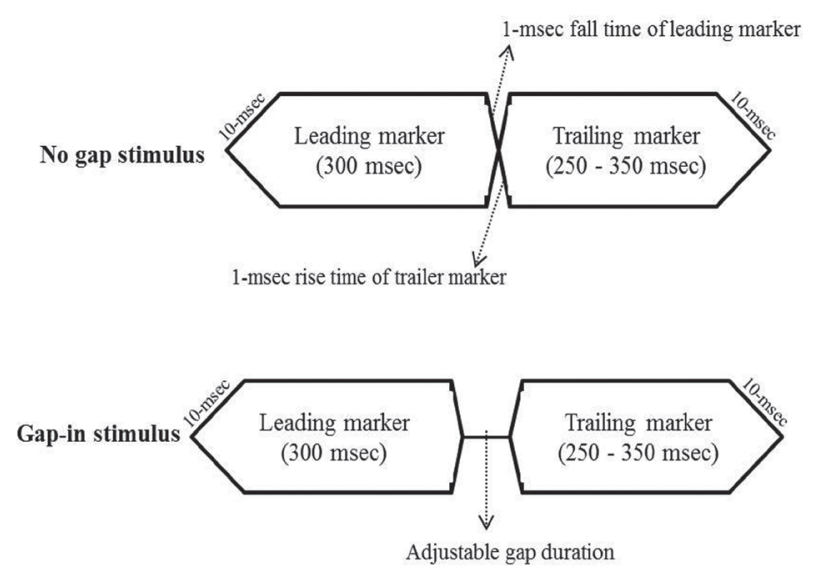

Figure 3 - Schematic representation of the transient durations in the no-gap and gap-in stimulus conditions. 
along with the mean and standard deviation values in a text file, which was saved on the hard drive of the computer for further analysis. The computed GDT for each task was the duration apart from the transients present in the standard stimulus. The Statistical Package for Social Sciences for Windows (Version 24.0, IBM Corp, Armonk, NY, USA) was used to analyze the data. A One-way repeated measure of Analysis of Variance (ANOVA), paired-samples t-test, and Pearson's correlation coefficient were performed.

Results. The computed GDT data from the last 4 reversals of each participant's responses under each stimulus condition across all subjects were averaged. The mean and SDs of the GDT for the BBN, NBN WC, and NBN AC conditions are provided in Figure 4 and Table 1.

Furthermore, individual GDTs for each participant in each condition are shown in Table 2. Mauchly's test indicated that the assumption of sphericity had been violated for the main effects of stimulus condition on GDTs $-\chi^{2}(2)=14.918, p<0.001$. As a result, degrees of freedom were corrected using Greenhouse-Geisser

Table 1 - Average gap detection thresholds in different stimulus conditions and corresponding standard deviations measured across subjects.

\begin{tabular}{lc}
\hline Stimulus condition & Mean \pm Standard deviation \\
\hline Broadband noise & $3.19_{ \pm} 0.72$ \\
Narrowband noise within-channel & $14.53_{ \pm} 6.85$ \\
Narrowband noise across-channel & $29.71_{ \pm} 13.8$ \\
\hline
\end{tabular}

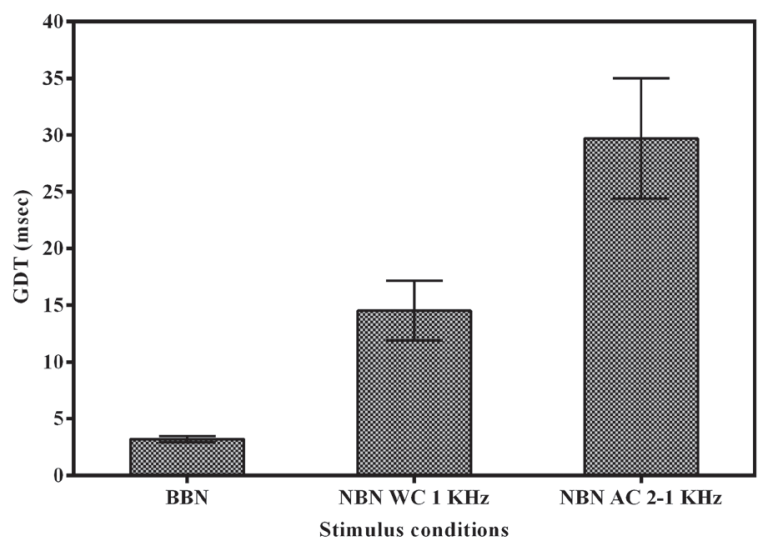

Figure 4 - Average gap detection thresholds (GDTs) along with standard errors (in msec) across stimuli conditions. Note: AC Stimulus condition is depicted as narrowband noise (NBN), leading marker frequency-trailing marker frequency in $\mathrm{KHz}$. estimates of sphericity (0.69) for the main effect. A one-way repeated measure of ANOVA indicated that the GDTs obtained differed significantly across the stimulus conditions (F $[1.38,35.88]=70.191$, $p<.0001$, eta $^{2}=0.73$ ). The Pairwise comparisons ( $\mathrm{t}$ tests) that was conducted, revealed significant differences among the GDTs obtained in different conditions. The analysis showed that the GDTs obtained with the NBN AC condition was significantly higher than those GDTs obtained with the NBN WC condition $(\mathrm{t}[26]=-6.012, p<0.0001])$ and also higher than the GDTs obtained with the BBN condition (t[26] $=-9.973, p<0.0001])$. Further, the analysis also showed that the GDTs obtained with the NBN WC condition was significantly higher than the GDTs obtained with the BBN condition $(\mathrm{t}[26]=-8.73$, $p<0.0001])$. Moreover, the Pearson correlation among the GDTs of the 3 stimulus conditions did not show any significant positive correlations among any pairs.

Table 2 - Individual gap detection thresholds (in msec) for the BBN, NBN WC and NBN AC stimulus conditions.

\begin{tabular}{|c|c|c|c|}
\hline Participant ID & BBN & NBN WC & NBN AC \\
\hline 1 & 3.0 & 20.7 & 37.4 \\
\hline 2 & 2.1 & 16.0 & 37.4 \\
\hline 3 & 2.8 & 21.4 & 20.4 \\
\hline 4 & 3.2 & 3.1 & 13.6 \\
\hline 5 & 3.0 & 18.9 & 27.3 \\
\hline 6 & 2.5 & 9.9 & 7.0 \\
\hline 7 & 3.9 & 9.8 & 20.6 \\
\hline 8 & 2.6 & 19.6 & 32.7 \\
\hline 9 & 3.8 & 15.8 & 24.0 \\
\hline 10 & 3.4 & 11.6 & 45.4 \\
\hline 11 & 3.9 & 27.3 & 19.4 \\
\hline 12 & 2.9 & 14.6 & 31.2 \\
\hline 13 & 5.5 & 18.3 & 32.7 \\
\hline 14 & 2.8 & 5.6 & 8.6 \\
\hline 15 & 3.0 & 9.7 & 20.7 \\
\hline 16 & 3.8 & 14.2 & 29.1 \\
\hline 17 & 4.4 & 11.9 & 20.6 \\
\hline 18 & 3.1 & 6.0 & 47.0 \\
\hline 19 & 3.2 & 20.6 & 51.9 \\
\hline 20 & 2.3 & 6.6 & 21.9 \\
\hline 21 & 3.1 & 19.6 & 47.1 \\
\hline 22 & 2.3 & 12.0 & 12.4 \\
\hline 23 & 3.6 & 9.3 & 11.9 \\
\hline 24 & 3.2 & 22.1 & 48.9 \\
\hline 25 & 2.8 & 4.8 & 50.7 \\
\hline 26 & 3.4 & 29.7 & 44.8 \\
\hline 27 & 2.5 & 13.1 & 37.4 \\
\hline
\end{tabular}


Discussion. The aim of the study was to explore the clinical utility of the Windows-based psychoacoustic experimental software Psycon (Version 2.18) and to estimate GDTs and to provide normative values for young listeners in the BBN, NBN WC, and NBN AC conditions. The overall results obtained with the above detailed parameters were consistent with those of previous study that used a similar methodology. ${ }^{7-9,11,14,21,23-25}$

In the study, the temporal acuity abilities measured with GDT using BBN revealed an average of $3.19 \mathrm{msec}$ silence detected by the young listeners. Similar findings obtained with ATTR have been reported by Lister et $\mathrm{al}^{10}$ for 30 adult listeners with normal hearing. They also reported an average GDT of $3.2 \mathrm{msec}$ (SD: $1.9 \mathrm{msec}$ ) with $\mathrm{BBN}$. In addition, our findings were consistent with Hoover et $\mathrm{al}^{7}$ who found an average of $4.53 \mathrm{msec}$ of silence obtained using the GIN test with 19 young normal hearing adults. In line with these findings, using the GIN test, Shinn et $\mathrm{al}^{26}$ have reported an average temporal gap of $4.87 \mathrm{msec}(\mathrm{SD}=1.25 \mathrm{msec})$ and $5 \mathrm{msec}$ $(\mathrm{SD}=1.16 \mathrm{msec})$ for right and left ears, respectively for 22 normal hearing listeners aged between 12 to 18 years. Similarly, using the GIN test, Samelli and Schochat ${ }^{27}$ have reported an average of $4.19 \mathrm{msec}$ temporal gap for BBN. Overall, our findings on GDTs show that Psycon (Version 2.18), along with appropriate AUX codes, could reliably provide accurate GDTs using BBN.

In contrast to the above findings based on the BBN stimulus, the average GDT using the NBN WC stimulus with a marker frequency of $1.0 \mathrm{KHz}$ revealed that the studied listeners detected an average silence of $14.53 \mathrm{msec}$. These findings were consistent with the outcome of Florentine's et $\mathrm{al}^{12}$ study. The midpoint of the psychometric function curve of $1.0 \mathrm{KHz}$ was $12.7 \mathrm{msec}$ (SD: $1.28 \mathrm{msec}$ ). Similarly, Formby et $\mathrm{a}^{28}$ have found an average GDT of $15.65 \mathrm{msec}$ for the leading $0.9 \mathrm{KHz}$ (bandwidth: $190 \mathrm{~Hz}$ ) marker and trailer marker of $0.99 \mathrm{KHz}$ (bandwidth: $100 \mathrm{~Hz}$ ), with a varying leading marker duration of 50-90 msec and a trailer marker duration of 100-140 msec. However, in the present study, the results (average GDT of $14.53 \mathrm{msec}$ ) obtained with the NBN WC stimulus were comparatively higher than the average $8.43 \mathrm{msec}$ reported by Phillips and Smith ${ }^{29}$ and the average $6.7 \mathrm{msec}$ reported by Elangovan and Stuart ${ }^{30}$ for similar NBN makers of $1.0 \mathrm{KHz}$. Also, the mean GDT $(14.53 \mathrm{msec})$ from the present study is higher than the mean GDT of 3.2 msec with different markers centered at $2 \mathrm{KHz}$ in Lister et al. ${ }^{10}$ These differences among studies could be due to the bandwidth of the signal considered, and the slope of the digital filters used for designing the stimulus. Furthermore, these differences also could be due to the procedure differences involved in the experiments.

In this study, the temporal resolution abilities assessed with respect to the AC paradigm revealed that an average of $29.71 \mathrm{msec}(\mathrm{sd}: 13.8 \mathrm{msec}$ ) silence duration was detected by the listeners. These findings were comparative with previous studies that involved similar marker frequencies of $2.0 \mathrm{KHz}$ as leading marker and $1.0 \mathrm{KHz}$ as trailer marker. ${ }^{14,21,23}$ For example, Lister et $\mathrm{al}^{21}$ have reported GDTs of $27 \mathrm{msec}$ in an anechoic listening environment and reported GDTs of $29.9 \mathrm{msec}$ in a reverberant listening environment. However, the obtained GDTs for the AC condition were lower than other investigations. For example, the obtained mean for GDTs was lower than the mean GDTs found by Hess et $\mathrm{al},{ }^{11}$ which was $35.71 \mathrm{msec}$ at $50 \mathrm{~dB}$ sensation level, and by Lister et al, ${ }^{10}$ which was $42.6 \mathrm{msec}$ obtained for dissimilar markers centered at $2 \mathrm{KHz}$ before the gap and one $\mathrm{KHz}$ (both of these studies used the ATTR instrument). Moreover, such variations between our findings and other study findings may be due to instrumentation and procedure differences.

In addition, the finding from the study of no correlation among the 3 conditions suggests that a separate auditory process may be involved WC and AC gap processing. The study of Phillips and Smith ${ }^{29}$ measured temporal gap processing in 95 normal hearing adults. They obtained gap detection thresholds for 2 NBN WC conditions with markers centered at $1.0 \mathrm{KHz}$ and $4.0 \mathrm{KHz}$, and for one NBN AC condition with markers centered at $4.0 \mathrm{KHz}$ and $1.0 \mathrm{KHz}$. They reported evidence for a weaker correlation between the WC and AC conditions compared to a higher correlation between the $2 \mathrm{WC}$ conditions, which suggests a separate process is involved in the identification of the gaps in WC and AC. ${ }^{29}$ According to Phillips and Smith, ${ }^{29,31}$ the temporal processing of WC gap detection task of same marker frequencies (spectrally overlapping) involves an activation of the same neuron regions in the peripheral auditory system. The silent gap is identified due to the detection of a lack of neural activity (a discontinuity detection process). In contrast, the temporal processing of the AC gap detection task of dissimilar makers (spectrally non-overlapping) involves an activation of different neuron regions in the peripheral auditory system. The silent gap is identified due to the monitoring of the time between the offset of the leading marker and the onset of the trailing marker (a relative timing process). The complexity of the relative timing process due to frequency disparity induces more time to identify 
the temporal gap, and hence larger GDTs. Thus, the SD obtained by the present study also supports the complexity effects of AC gap processing in which higher variations exist in AC temporal acuity compared to WC among normal hearing listeners, which is consistent with the previous studies. . $^{14,21,23}$

The overall findings of the study indicate that temporal resolution abilities using gap detection could be reliably and easily assessed using computer applications with appropriate stimulus and experimental parameters. However, the findings from the study were limited to $1 \mathrm{KHz}$ for WC and $2-1 \mathrm{KHz}$ AC gap investigations. Further, the variability in the GDTs of the WC and AC conditions across studies suggest a need exists for more studies on standardized procedures to obtain normative data on GDTs, and also to create clinically reliable tools to assess the temporal resolution of speech and to diagnose CAPD.

Acknowledgment. The authors appreciate the support from the Research Center, College of Applied Medical Sciences and the Deanship of Scientific Research, King Saud University, Riyadh, Kingdom of Saudi Aarabia.

\section{References}

1. American Speech-Language-Hearing Association. Central auditory processing: current status of research and implications for clinical practice. American JournalAudiology 1996; 5: 41-52.

2. Reed CM, Braida LD, Zurek PM. Review of the literature on temporal resolution in listeners with cochlear hearing impairment: a critical assessment of the role of suprathreshold deficits. Trends Amplif 2009; 13: 4-43.

3. Lister J, Roberts R, Shackelford J, Rogers C. An adaptive clinical test of temporal resolution. Am J Audiol 2006; 15: 133-140.

4. Musiek FE, Shinn JB, Jirsa R, Bamiou DE, Baran JA, Zaida E. GIN (gaps-in-noise) test performance in subjects with confirmed central auditory nervous system involvement. Ear Hear 2005; 26: 608-618.

5. Keith R. Random gap detection test. St Louis (MO): Auditec; 2000

6. Kwon BJ. AUX: a scripting language for auditory signal processing and software packages for psychoacoustic experiments and education. Behavior Ressearch Methods 2012; 44: 361-373.

7. Hoover E, Pasquesi L, Souza P. Comparison of clinical and traditional gap detection tests. J Am Acad Audiol 2015; 26: 540-546.

8. Wong AC, McPherson B. Adaptive tests of temporal resolution: comparison with the gaps-in-noise test in normal-hearing young adults. Int J Audiol 2015; 54: 29-36.

9. Phillips DP, Hall SE, Harrington IA, Taylor TL. "Central" auditory gap detection: a spatial case. J Acoust Soc Am 1998; 103: 2064-2068.

10. Lister J, Roberts R, Krause JC, DeBiase D, Carlson H. An adaptive clinical test of temporal resolution: within-channel and across-channel gap detection. Int J Audiol 2011; 50: 375-384.
11. Hess BA, Blumsack JT, Ross ME, Brock RE. Performance at different stimulus intensities with the within- and across-channel adaptive tests of temporal resolution. Int J Audiol 2012; 51: 900-905.

12. Florentine M, Buus S, Geng W. Psychometric functions for gap detection in a yes-no procedure. The Journal of the Acoustical Society of America 1999; 106: 3512-3520.

13. Grose JH, Hall JW 3rd, Buss E, Hatch D. Gap detection for similar and dissimilar gap markers. J Acoust Soc Am 2001; 109 : 1587-1595.

14. Lister J, Besing J, Koehnke J. Effects of age and frequency disparity on gap discrimination. J Acoust Soc Am 2002; 111: 2793-2800.

15. Mishra SK, Panda MR, Herbert C. Enhanced auditory temporal gap detection in listeners with musical training. J Acoust Soc Am 2014; 136: EL173-EL178.

16. Mishra SK, Panda MR. Rapid auditory learning of temporal gap detection. J Acoust Soc Am 2016; 140: EL50.

17. Mishra SK, Dey R, Davessar JL. Temporal resolution of the normal ear in listeners with unilateral hearing impairment. $J$ Assoc Res Otolaryngol 2015; 16: 773-782.

18. Boersma P, Weenink D. Praat: doing phonetics by computer. 6.0.14. [Updated 2018 March 14]. Available from: http://www. praat.org/

19. Lister J, Tarver K. Effect of age on silent gap discrimination in synthetic speech stimuli. J Speech Lang Hear Res 2004; 47: 257-268.

20. Dai H, Micheyl C. Separating the contributions of primary and unwanted cues in psychophysical studies. Psychol Rev 2012; 119: 770-788.

21. Lister J, Koehnke JD, Besing JM. Binaural gap duration discrimination in listeners with impaired hearing and normal hearing. Ear Hear 2000; 21: 141-150.

22. Brown LG. Additional rules for the transformed up-down method in psychophysics. Percept Psychophys 1996; 58: 959-962.

23. Lister J, Roberts R, Lister FL. An adaptive clinical test of temporal resolution: age effects. Int J Audiol 2011; 50: 367-374.

24. Buss E, Hall III JW, Porter H, Grose JH. Gap detection in school-age children and adults: effects of inherent envelope modulation and the availability of cues across frequency. $J$ Speech Lang Hear Res 2014; 57: 1098-1107.

25. Hall JW, Buss E, Ozmeral EJ, Grose JH. The effect of noise fluctuation and spectral bandwidth on gap detection. J Acoust Soc Am 2016; 139: 1601-1610.

26. Shinn JB, Chermak GD, Musiek FE. GIN (gaps-in-noise) performance in the pediatric population. J Am Acad Audiol 2009; 20: 229-238.

27. Samelli AG, Schochat E. The gaps-in-noise test: Gap detection thresholds in normal-hearing young adults. Int J Audiol 2008; 47: 238-245.

28. Formby C, Barker C, Abbey H, Raney JJ. Detection of silent temporal gaps between narrow-band noise makers having second-formant like properties of voiceless stop/vowel combinations. J Acoust Soc Am 1993; 93: 1023-1027.

29. Phillips DP, Smith JC. Correlations among within-channel and between-channel auditory gap-detection thresholds in normal listeners. Perception 2004; 33: 371-378.

30. Elangovan $S$, Stuart A. Natural boundaries in gap detection are related to categorical perception of stop consonants. Ear Hear 2008; 29: 761-774.

31. Phillips DP. Auditory gap detection, perceptual channels, and temporal resolution in speech perception. J Am Acad Audiol 1999; 10: 343-354. 